\title{
Editorial
}

\section{Recognizing and Reacting to challenges}

\author{
Deborah Gaebler-Spira $^{\mathrm{a}, *}$ and Michael Green ${ }^{\mathrm{b}}$ \\ ${ }^{a}$ Feinberg Northwestern School of Medicine Department of Physical Medicine and Rehabilitation Shirley Ryan \\ Ability Lab, Chicago, IL, USA \\ ${ }^{\mathrm{b}}$ University of Utah Health, Salt Lake City, UT, USA
}

Welcome to the third special Cerebral Palsy (CP) focused issue of the Journal of Pediatric Rehabilitation Medicine (JPRM). Mike and I are delighted to have such a volume of high-quality work to present this year. As promised, this annual special issue includes Needle Tips, Hot Topics, and human-TIES, along with original articles on topics related to $\mathrm{CP}$.

This year's Needle Tips include two selections. The first article uses a case-based approach to discuss consideration of a child who is a non-responder emphasizing a precision medicine perspective in the context of current rehabilitation practice. We hope this case-based approach is helpful and well received. If you have cases that are of interest or if you would like to send us a challenge to confuse the gurus... We welcome those for future issues!

The second selection comes as a cautionary reminder of the interface between medicine and the law. This topic is rarely discussed but is an unfortunate potential reality for injectors who use neurotoxins in vulnerable populations. The article explores the discussion with two scenarios that led to encounters with the legal system. The author highlights key issues of safe patient practices, and then proceeds with a practical discussion of the legal process. Everyone is mindful of the negative impact that litigation has on the entire health care system, so we thank the authors for including important

\footnotetext{
*Corresponding author: Deborah Gaebler-Spira, Feinberg Northwestern School of Medicine Department of Physical Medicine and Rehabilitation Shirley Ryan Ability Lab, Chicago, IL, USA. E-mail: dgaebler@ric.org.
}

information on protecting the injector and preventing risk to the injectors' practice and their patients.

The Hot topics section is focused on healthcare disparity, embedded racism, and two important ways to mitigate the continued problems of exclusion of disadvantaged populations. We all witness the impact this has on our communities with the emergence of COVID19. Disparity is equally present and true for those with chronic conditions. We must not turn away! The lead commentary is a summary of the known health care disparities written to provide the context of the current landscape. We anticipate this will be a helpful way to focus our attention as we work to equalize care.

One approach to reducing healthcare disparities is to improve online information about access to high quality information about CP for everyone to improve and encourage shared decision making. Most of the world population is now comfortable with smart phone use and internet access, therefore, equal access to accurate information is critical. This open access to information is a mixed blessing as we have witnessed in the past few years. The Cerebral Palsy Foundation (CPF) has a dedicated mission to provide information that can be a benchmark for resource use. The senior vice president of strategic partnerships at the CPF authors a commentary and provides resource options to improve communication with our patients and their families. We hope you are able to use these resources to learn, teach and encourage your patients and families to participate fully in their care.

The next article highlights the efforts of the Cerebral Palsy Research Network (CPRN). We have asked the 
leadership of CPRN to provide updates on the registry. We believe the information gained from the CPRN has the potential to decrease disparity by examining practice variations and analyzing outcomes. For those of you lucky enough to participate in this network, we applaud your hard work and support your involvement. For many of us who do not easily integrate with the electronic medical records system via EPIC, or who are outside of a large institutional network, it is important to understand this incredible multisite informatics registry effort. Hopefully, all of us will be able to participate at some level in the big data research and discussions. This network is an ideal platform that can equalize care through implementation strategies and spotlight issues related to healthcare disparities.

We are welcoming two additional guest editors for 2022. Heakyung Kim, an internationally known leader in Pediatric Rehabilitation Medicine (PRM) and in the care of children with $\mathrm{CP}$, and Matthew MacCarthy, an emerging leader in the field of PRM. We are moving the JPRM Cerebral Palsy Special Issue in the future to the first issue of the year starting in 2022 (15.1), to coincide with the annual March CP awareness month. Please join us in submitting, reviewing, and providing support and content for the $2022 \mathrm{CP}$ issue.
Our last CP issue went to press as COVID-19 swept over the world. This past year upended so much of our in-person contact at work and in meetings along with affecting time with family. However, we see the emergence of many virtual communities forming to support professional education, research, clinical care, and stakeholder engagement. This is an era of cooperation and collaboration!

We have faced and are tackling many difficult problems, of which we present just a few. Now as we round the corner of isolation to vaccination against COVID19, there is light at the end of the tunnel. We want to acknowledge an enormous talent pool of problem solvers emerging in the field of Pediatric Rehabilitation Medicine. The PRM field faces difficult problems, and by working together with our colleagues, patients and their parents, we all create a stronger, more inclusive model and world for the children and adults with CP.

Deb and Mike Guest-Editors

"Not everything can be changed that is faced, but nothing can be changed until it is faced." James Baldwin 